\title{
Advanced Techniques in Biomedical Optical Imaging
}

\author{
Nanguang Chen, ${ }^{1}$ Baohong Yuan, ${ }^{2}$ and Javier A. $\mathbf{J o}^{3}$ \\ ${ }^{1}$ Department of Bioengineering, National University of Singapore, 7 Engineering Drive 1, Singapore 117574 \\ ${ }^{2}$ Bioengineering Department, University of Texas at Arlington, 500 UTA BLVD, Arlington, TX 76010, USA \\ ${ }^{3}$ Department of Biomedical Engineering, Texas A\&M University, 3120 TAMU, College Station, TX 77843-3120, USA
}

Correspondence should be addressed to Nanguang Chen, biecng@nus.edu.sg

Received 4 March 2012; Accepted 4 March 2012

Copyright (C) 2012 Nanguang Chen et al. This is an open access article distributed under the Creative Commons Attribution License, which permits unrestricted use, distribution, and reproduction in any medium, provided the original work is properly cited.

Numerous novel optical imaging techniques have been developed in the past decade for widespread basic biological research and clinical applications. While optical microscopy remains as an indispensable tool for high-resolution cellular or subcellular imaging, a larger imaging depth achieved with scattered light is becoming more and more attractive for noninvasive imaging of large human organs. For example, diffuse optical tomography (DOT) has been extensively investigated for potential applications in breast cancer detection, brain function study, and small animal whole-body imaging. Optical coherence tomography (OCT) is also one of hot research areas. Besides its successful application in ophthalmology, OCT is expected to play an increasingly important role in blood vessel evaluation and skin cancer diagnosis.

Four out of seven papers published in this special issue are related to optical imaging with scattered light. Image reconstruction is an integrated component of DOT. B. Wu et al. simplified the reconstruction process to a blind source separation (BSS) problemin their paper entitled "Diffuse optical imaging using decomposition methods." Three matrix decomposition methods (Independent Component Analysis, Principal Component Analysis, and Nonnegative Matrix Factorization) were used to localize targets embedded in a turbid medium. The targets were considered as uncorrelated or independent virtual sources that led to perturbations in the detected diffusive light intensity. While these methods do not directly provide tomographic images, the high accuracy in localizing small heterogeneities may be valuable for detecting tumors in their early stages.

Z.-J. Lin et al. reported a CCD-based DOT system for volumetric imaging of small animals in their paper entitled "Volumetric diffuse optical tomography for small animals using a CCD-camera-based imaging system." With a 12-bit CCD camera, the optical signal from regions up to $20 \mathrm{~mm}$ in depth can be detected by pixel binning. In addition, a depth compensation algorithm is integrated in their system for improved image quality. Because of its simplicity, low-cost, and portability of such a system, a CCD-camera-based DOT may become a highly appropriate imaging tool for preclinical studies involving small animals. For example, their approach may be used to localize and evaluate tumors embedded in a small animal.

Diabetes is one of major global health problems, which affects almost one-fifth of the world population. Selfmonitoring of blood glucose is critical for maintaining the health of diabetic patients. E. Nakamachi et al. developed a 3D blood vessel search system combined with an automatic blood sampling system, which were integrated into a pointof-care system for medical care in their paper entitled "Development of automatic 3D blood vessel search and automatic blood sampling system by using hybrid stereo-autofocus method." They adopted the hybrid stereo-autofocus method to detect the 3D location of blood vessels. When detecting a $0.5 \mathrm{~mm}$ diameter blood vessel embedded in the depth range of $0.5-2.5 \mathrm{~mm}$, the localization precision was $63 \mu \mathrm{m}$ on average. The combined system was evaluated with phantoms to demonstrate its potential in real medical applications.

Light scattering from biological cells has been used as a diagnostic tool for diseases in superficial tissues. Simulating the scattering process is very important for understanding and predicting the scattering patterns. S. Saltsberger et al. presented an analytical model of a multilayer spherical scattering cell in their paper entitled "Multilayer Mie scattering model for investigation of intracellular structural changes in the nucleolus and cytoplasm." They developed and implemented a multilayer Mie-like model to treat the scattering of light from biological cells, whose internal structures are spherically symmetric. It was shown that the multilayer model provided 
similar results as the finite-difference time-domain (FDTD) method, which is more computationally expensive.

Combining optical imaging with other image modalities, such as ultrasound, X-ray, and MRI, has attracted considerable interest recently. For example, the field of photoacoustic imaging has been growing rapidly in the past few years. Y. Liu et al. investigated a different approach to combine fluorescence imaging with ultrasound, which offers better penetration and spatial resolution in their paper entitled "Effect of fluorescent particle size on the modulation efficiency of ultrasound-modulated fluorescence." They studied the effect of fluorescent particle size on the modulation depth of ultrasound modulated fluorescence. According to their results, there was a moderate twofold increase in the modulation depth when the fluorescent particle size increased from $5 \mathrm{~nm}$ to $1 \mu \mathrm{m}$. The authors suggested that other approaches should be explored to significantly improve the modulation efficiency.

While the basic principle of optical coherence tomography (OCT) is straightforward, there is a continuous drive to search for the optimal implementations. Swept source optical coherence tomography is the proven choice of higher imaging speed and better sensitivity. S. Chang et al. proposed a method to reconstruct OCT images from the combined spectral data obtained from two swept sources in their paper entitled "Dual-source swept-source optical coherence tomography reconstructed on integrated spectrum." It was demonstrated that their method resulted in OCT images with richer spectral and resolution information than any of the individually reconstructed OCT images. This method may be generalized for a swept source OCT system with multiple swept sources.

It is exciting to see that optical imaging methods start to play a role in radiation therapy, which uses Magavoltage (MV) X-ray beams to kill cancer cells. A critical issue in radiation therapy is how to guide the radiation dose towards the tumor while minimizing the harm to the surrounding normal tissues. A. Teymurazyan and G. Pang proposed a novel design of MV X-ray detectors for accurate locating the target in the treatment room before the start of radiation therapy in their paper entitled "Megavoltage X-ray imaging based on Cerenkov effect: A new application of optical fibres to radiation therapy." Their design was based on the Cerenkov effect. The proposed detector consists of a matrix of optical fibres aligned with the incident X-rays and coupled to an active matrix flat panel imager (AMFPI) for image readout. X-ray energy absorbed in optical fibres is converted into visible light for improved detection sensitivity. Monte Carlo simulation was used to study imaging and dosimetric characteristics of such a detector. The simulation results showed that the proposed detector had a zero-frequency detection quantum efficiency more than an order of magnitude higher than that of current electronic portal imaging device (EPID) systems, while achieving a spatial resolution comparable to that of video-based EPIDs. The proposed detector is also less sensitive to scattered X-rays from patients than existing EPIDs.

\footnotetext{
Nanguang Chen Baohong Yuan Javier A. Jo
} 

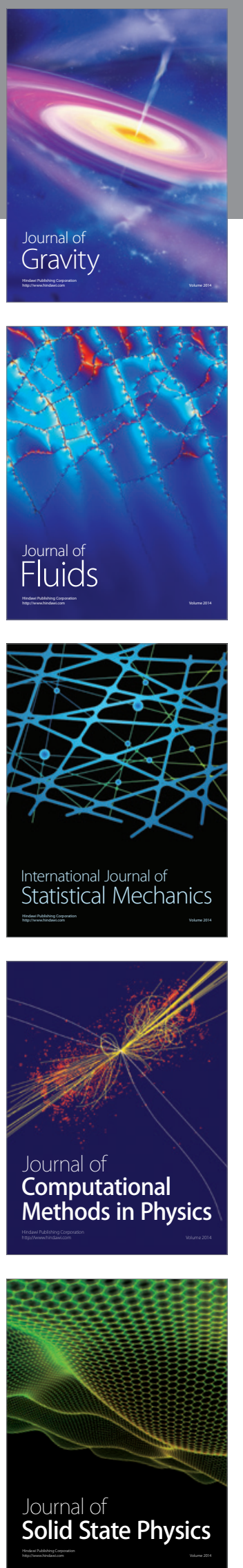

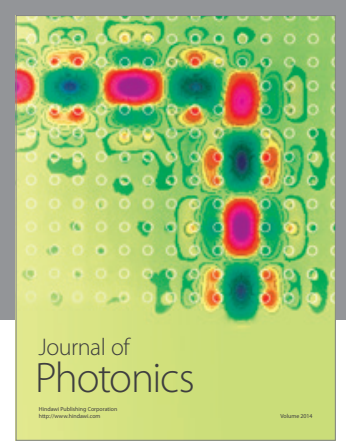

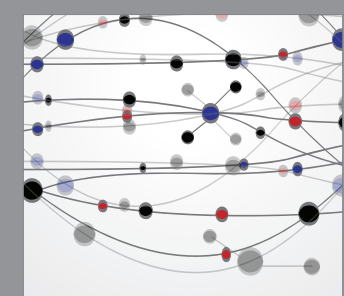

The Scientific World Journal
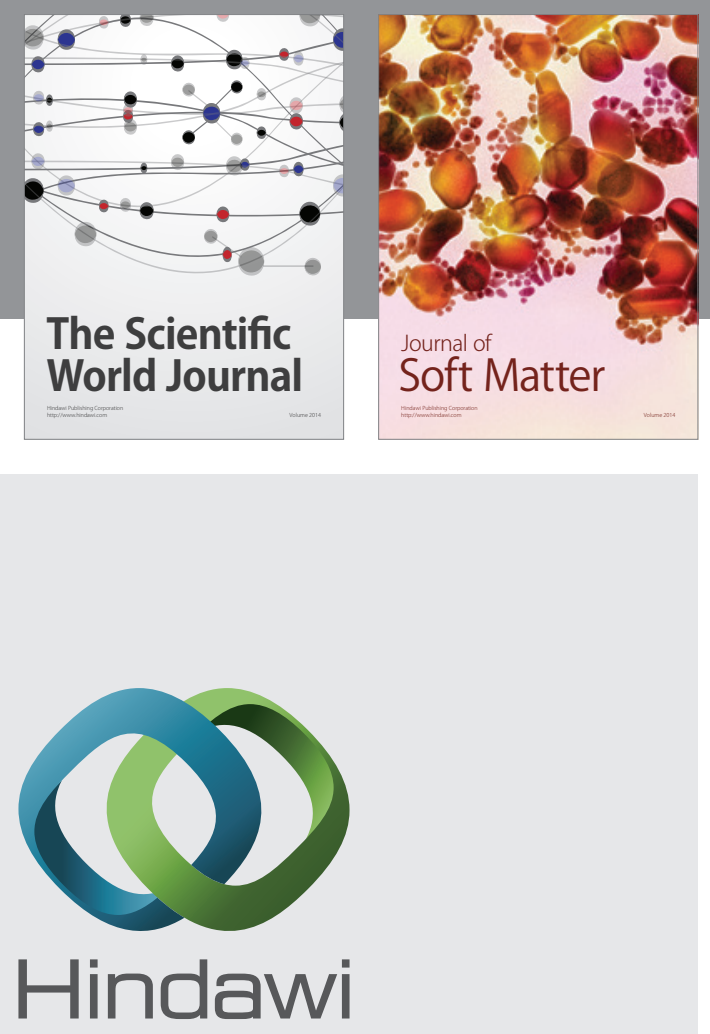

Submit your manuscripts at

http://www.hindawi.com
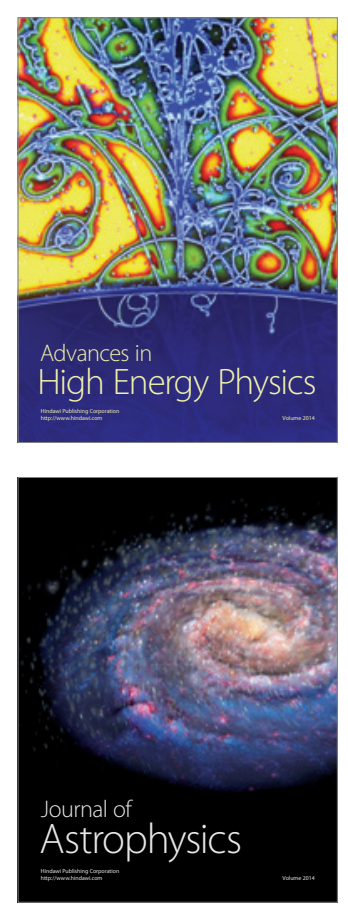
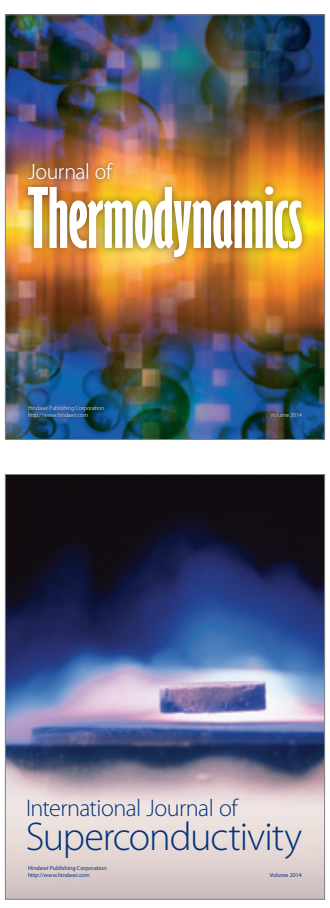
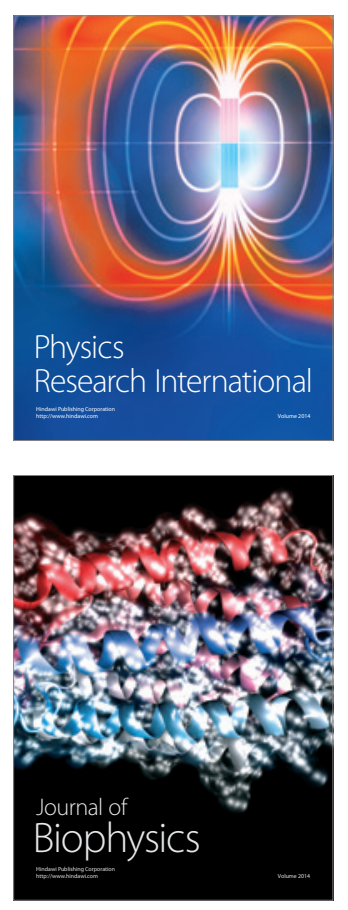
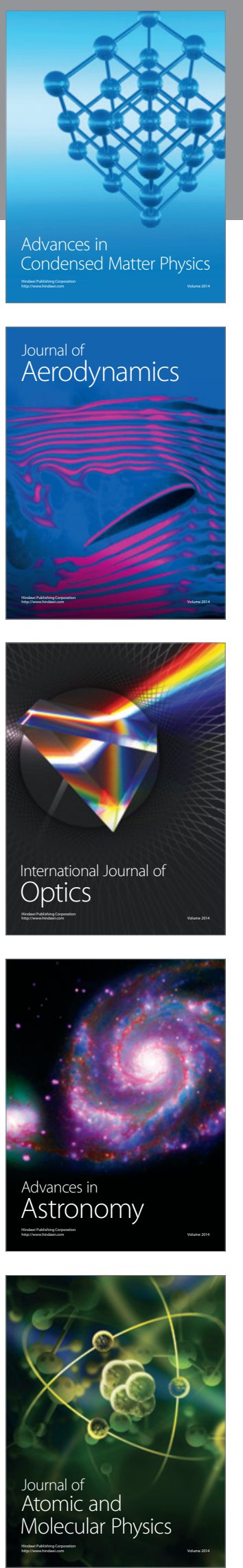